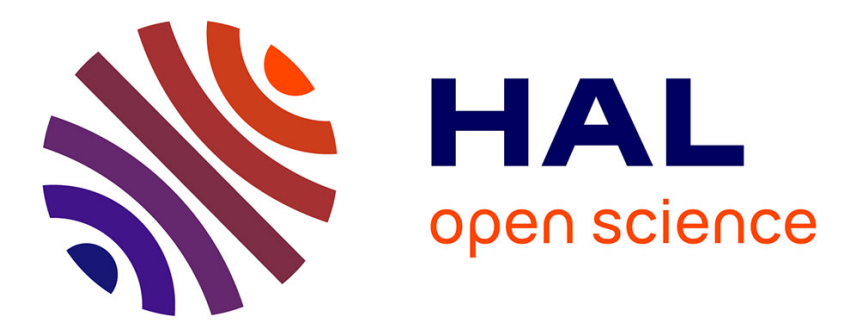

\title{
Le raisonnement juridique dans tous ses états Pierre Brunet
}

\section{- To cite this version:}

Pierre Brunet. Le raisonnement juridique dans tous ses états. Droit et Société, 2013, 1 (83), pp.193202. 10.3917/drs.083.0193 . halshs-00806365

\section{HAL Id: halshs-00806365 https://shs.hal.science/halshs-00806365}

Submitted on 2 Jul 2013

HAL is a multi-disciplinary open access archive for the deposit and dissemination of scientific research documents, whether they are published or not. The documents may come from teaching and research institutions in France or abroad, or from public or private research centers.
L'archive ouverte pluridisciplinaire HAL, est destinée au dépôt et à la diffusion de documents scientifiques de niveau recherche, publiés ou non, émanant des établissements d'enseignement et de recherche français ou étrangers, des laboratoires publics ou privés. 


\section{Le raisonnement juridique dans tous ses états}

Texte paru dans Droit et Société, 2013/1, n. 83, p. 193-202

\section{Pierre Brunet}

Centre de Théorie et Analyse du Droit (CTAD), Université Paris Ouest Nanterre-La Défense, 200 avenue de la République, F-92001 Nanterre cedex

< pierre.brunet@u-paris10.fr >

\section{À propos de...}

- Alexander Larry et Sherwin Emily, Demystifying Legal Reasoning, Cambridge : Cambridge University Press, 2008, $253 \mathrm{p}$.

- SCHAUER Frederick, Thinking Like A Lawyer. A New Introduction To Legal Reasoning, Cambridge : Harvard University Press, 2009, 239 p.

À l'heure où les juristes français réfléchissent au moyen de transmettre leur savoir, de réorganiser leurs enseignements, de s'émanciper d'une vision institutionnelle de la discipline que d'aucuns jugent désuète, il n'est peut être pas inutile de faire un pas de côté et de se pencher sur quelques écrits récents relatifs à la question délicate mais toujours actuelle du raisonnement juridique en général et plus particulièrement de la forme qu'il prend et des contraintes qu'il rencontre dans les États qui sont les nôtres aujourd'hui, les États dits constitutionnels.

Les deux ouvrages dont il sera question ici abordent donc un même thème en empruntant des points de vue très différents. La question y est de savoir si le raisonnement juridique présente une spécificité quelconque, et si oui laquelle. Larry Alexander et Emily Sherwin, pour leur part, apportent une réponse négative dépourvue d'ambiguïté, radicalisant quelque peu certaines positions prises par Frederick Schauer dans des travaux antérieurs 1 . Ce dernier, quant à lui, défend, comme à son habitude, une position nettement plus nuancée et qui fait preuve d'un certain raffinement, voire d'un raffinement certain.

1. On pense à Frederick Schauer, Playing by the Rules: A Philosophical Examination of Rule-Based Decision-Making in Law and in Life, Oxford: Oxford University Press, 1991, not. p. 183-187 sur les précédents. Notons au passage que F. Schauer et L. Alexander se connaissent très bien et ont, par le passé, écrit ensemble, voir not. : Frederick SchaUer et Larry ALEXANDER, " Law's Limited Domain Confronts Morality's Universal Empire », William and Mary Law Review, 48 (5), 2007, p. 1579-1603 et ID., « On Extrajudicial Constitutional Interpretation », Harvard Law Review, 110 (7), 1997, p. 1359-1387.

Texte paru dans Droit et Société, 2013/1, n. 83, p. 193-202 


\section{Le raisonnement juridique sans spécificité}

La thèse de L. Alexander et $\mathrm{E}$. Sherwin est, dès le départ, annoncée comme très simple 2 : le raisonnement juridique n'a aucune spécificité, il est un raisonnement ordinaire appliqué à des problèmes juridiques et ceux qui doivent décider juridiquement se trouvent engagés soit dans un raisonnement moral, soit dans un raisonnement empirique soit encore dans une déduction à partir de règles obligatoires ou encore "sérieuses ». L. Alexander et E. Sherwin contestent fortement que les solutions passées puissent déterminer les solutions futures, que des analogies soient possibles, que les principes juridiques soient logiquement cohérents ni « normativement attractifs », et ils ne croient pas non plus que la décision juridique conduise celui qui la prend à adopter des modes spécifiques d'interprétation des textes.

Pour autant, leur démonstration ne suit pas du tout la voie indiquée par les juristes réalistes, tel Karl N. Llewellyn, pour qui « la » doctrine du précédent est un Janus qui permet aussi bien de se débarrasser d'une solution antérieure que de l'utiliser en tant que de besoin, de sorte que le juge peut faire exactement ce qu'il veut selon la façon dont il découpe les cas 3 .

L'analyse que livrent L. Alexander et E. Sherwin repose sur une certaine conception de la fonction du droit, de la règle, et de la fonction même du juge. Selon les auteurs, en effet, le besoin de raisonnement juridique se fait sentir lorsque les membres d'une communauté, qui se sont mis d'accord sur des valeurs morales à un assez haut niveau de généralité et qui acceptent ces valeurs comme des guides pour leur propre action, se trouvent en désaccord sur les conséquences des valeurs morales auxquelles ils adhèrent ou lorsqu'ils sont incertains quant aux modalités permettant de les réaliser. Pour réduire le coût moral des divergences et de leur incertitude, ils peuvent déléguer le pouvoir de trancher à une autorité choisie par eux. Par " trancher », L. Alexander et E. Sherwin entendent non pas seulement le pouvoir de choisir une solution mais aussi le fait de raisonner, de délibérer, et de justifier un choix. Les décisions prises ne seront peut-être pas moralement justes mais au moins seront-elles argumentées et ne pourront faire seulement appel à l'intuition. Enfin, compte tenu de ce que l'autorité en question ne peut être convoquée à chaque controverse, la communauté imaginée par $L$. Alexander et $E$. Sherwin aura besoin de règles obligatoires (" authoritatives »), de règles sérieuses, compréhensibles et rédigées en termes relativement univoques.

Ainsi n'adhèrent-ils nullement au scepticisme des réalistes à l'égard des règles. Pour eux, une règle est en mesure de déterminer sa propre application et une règle est sérieuse précisément lorsqu'elle est en mesure de déterminer son application à un cas particulier. De même, ils n'estiment pas nécessaire de définir le droit et refu-

2. Geoffrey Samuel s'est employé à en montrer les faiblesses en s'appuyant sur une solide bibliographie reposant sur des travaux de sciences sociales quasi exclusivement francophones afin de montrer que le raisonnement juridique ne consiste pas seulement en opérations à partir de et à propos des règles, mais qu'il doit aussi prendre en compte des faits et la construction des situations de fait: Geoffrey SAmuEL, "Can Legal Reasoning Be Demystified? », Legal Studies, 29 (2), 2009, p. 181-210.

3. Voir Karl Nickerson Llewellyn, The Bramble Bush. The Classic Lectures on the Law and Law School [1930], Oxford : Oxford University Press, 2008 [Steve Sheppard (ed.)], p. 68-71.

Texte paru dans Droit et Société, 2013/1, n. 83, p. 193-202 
sent de prendre parti dans le débat entre positivistes et jusnaturalistes sur la nature du droit. Selon eux, les deux positions sont complémentaires et non antinomiques et décrivent deux facettes différentes du droit ${ }^{4}$. Ils expliquent que toutes les règles sérieuses s'accompagnent d'un « dilemme » qui vient du fossé qui existe entre les buts poursuivis par celui qui pose une règle et le destinataire qui dispose toujours d'un jugement moral et critique à l'égard de la règle. C'est ce dilemme qui rend la position positiviste fragile même si, pour rendre compte du raisonnement juridique, les auteurs adoptent un point de vue détaché, volontiers associé au positivisme, en ce qu'ils privilégient la façon dont les juges réagissent lorsqu'ils sont confrontés au droit posé par une règle et à la façon dont ces mêmes juges distinguent entre le raisonnement à partir des règles posées et le raisonnement en l'absence de règles posées.

Leur thèse est que, selon le cas, soit les juges raisonnent par déduction - lorsqu'ils sont confrontés à des règles posées par d'autres qu'eux -, soit ils posent euxmêmes le droit, en se fondant sur un jugement moral et empirique, comme doit le faire n'importe quel créateur du droit. Ils distinguent ainsi deux modèles - et deux seulement - du raisonnement en common law (ils ne s'intéressent pas aux systèmes de droit codifié). Selon le premier modèle - le modèle qu'ils appellent naturel -, ce sont les cours qui décident de la meilleure solution, à l'aide d'un raisonnement moral (l'équilibre réflexif de Rawls) et d'un raisonnement empirique. Selon le second modèle - le modèle dit des règles -, les cours prennent au sérieux les règles utilisées par les cours précédentes et n'y renoncent que si ces mêmes règles ne fournissent pas de réponse. La différence entre les deux modèles réside dans la façon de considérer les décisions judiciaires et notamment celles passées : dans le premier modèle, elles sont des faits à propos du monde extérieur, elles ne sont pas obligatoires, elles peuvent être pertinentes dans la mesure où elles ont généré des attentes en termes de cohérence et de sécurité juridique, tandis que dans l'autre modèle, les décisions sont des sources de droit : elles sont obligatoires et les mettre en œuvre consiste en un raisonnement interprétatif et déductif.

Quels que soient les défauts de ces modèles, L. Alexander et E. Sherwin affirment qu'il n'y en a pas d'autres. Ce faisant, ils en rejettent deux autres très fréquemment mis en avant dans le système de common law : le modèle du raisonnement par analogie et le raisonnement fondé sur les principes. II n'y a donc pas de moyen terme tel que les juges découvriraient le droit dans les décisions passées ou des textes antérieurs, ou combineraient moralité et droit positif afin de construire des principes juridiques.

\section{La critique de l'analogie et la question du précédent}

Sans être d'une originalité extrême, leur critique de l'analogie mérite d'être relevée : ils reconnaissent sans difficultés que les juges ont (pris) l'habitude de chercher des cas similaires et des principes généraux communs, et si cette habitude joue

4. Pour une plus ample démonstration, voir leur précédent livre, Larry ALEXANDER et Emily SHERWIN, The Rule of Rules. Morality, Rules, and the Dilemmas of Law, Durham : Duke University Press, 2001.

Texte paru dans Droit et Société, 2013/1, n. 83, p. 193-202 
certes un rôle pour le développement du common law, elle ne constitue pas une forme de raisonnement spécifique - en d'autres termes, chercher les faits est une chose, raisonner avec eux en est une autre. Reste que la thèse de l'analogie, comme celle des principes juridiques communs, emporte un incontestable succès qui tient à plusieurs facteurs. Le précédent peut apparaître comme une source de contrainte du jugement sans pour autant jamais s'y substituer complètement ni annihiler la part constructive dont le juge de common law souhaite bénéficier. Cette contrainte peut elle-même résider, d'une part, dans les faits de l'espèce - ils sont identiques et donc doivent être soumis à la même règle -, d'autre part, dans les finalités poursuivies - la solution précédente était justifiée par la recherche de telle finalité, or tel cas exige la même finalité, donc la règle utilisée dans le cas précédent doit être appliquée. Enfin, le précédent permet de procéder au "distinguishing » bien connu des juristes de common law qui, expliquent les auteurs, est le revers de la médaille de l'argument a fortiori et justifie que ne soit pas appliquée une règle issue d'un cas précédent au motif que les faits du précédent invoqué ne sont pas les mêmes. Bref, l'analogie que l'on peut tirer d'un précédent peut parfaitement justifier cette rationalité spécifique du droit à laquelle sont attachés de nombreux juristes de common law et ce, depuis les propos mémorables de Coke ${ }^{5}$. Mais nos auteurs n'y croient pas une seconde. Selon eux, "ce ne sont ni l'analogie ni ces principes qui décident du cas » (p. 66) et « l'analogie n'a aucun pouvoir de contrainte» (p. 70).

Autrement dit, on ne raisonne pas «par analogie », laquelle n'est pas une méthode de raisonnement spécifique mais rien d'autre qu'une déduction qui ne dit pas son nom. Dans tous les cas, les juges qui prétendent s'en tenir aux analogies doivent nécessairement se fonder sur une règle générale de laquelle ils déduiront la solution particulière : il leur faudra déterminer les faits pertinents, choisir un critère de pertinence, décider de retenir celui-ci plutôt que celui-là... À chaque fois, il leur faudra passer par la médiation d'une règle générale. Et si d'aventure, une cour invoquait l'absence de toute règle antérieure pour justifier un choix en termes purement factuels, on ne pourrait que conclure à l'arbitraire pur et simple de sa décision car les faits, par eux-mêmes, ne décident de rien. Bref, de deux choses l'une, ou bien le « raisonnement par analogie » n'est qu'une forme cachée du raisonnement déductif, ou bien il n'est pas un raisonnement du tout et se réduit à une pure pétition de principe.

Cette thèse se heurte toutefois à une objection : toutes les analogies ne sont pas des précédents. C'est à cette distinction qu'est consacré le chapitre 5 du dernier (et très riche) livre de Frederick Schauer. L'argument se présente ainsi : l'analogie, c'est la situation dans laquelle un juge prend sa décision sur le seul fondement d'une solution antérieure au motif que cette dernière lui semble "bonne »; le précédent, lui, consiste pour un juge à appliquer une solution retenue par le juge supérieur au

5. Cf. Sir Edward COKE, The First Part of the Institutes of the Law of England (Coke upon Littleton) [1628], réimpression: The Selected Writings of Sir Edward Coke, volume 2 [1639], Indianapolis : Liberty Fund 2003 : "Reason is the life of the Law, nay the common law itself is nothing else but reason, which is to be understood of an Artificial perfection of reason, gotten by long study, observation, and experience, and not of every man's natural reason. »

Texte paru dans Droit et Société, 2013/1, n. 83, p. 193-202 
motif qu'il est juridiquement soumis à cette solution. On doit d'ailleurs distinguer deux types de précédents : celui vertical - une cour inférieure est censée faire application de la règle elle-même appliquée par une cour supérieure dans un cas semblable -, et celui horizontal - une même cour est censée appliquer la même règle à deux cas semblables qui se présentent successivement. Du point de vue juridique, décider à partir d'un précédent ne consiste pas seulement à prendre une solution identique en considérant que les faits sont très proches, semblables voire en tous points identiques. Une cour raisonne à partir d'un précédent, explique F. Schauer, lorsqu'elle se conforme à une décision antérieure soit parce que cette décision émane d'une cour supérieure soit encore, dans le cas d'un précédent horizontal, parce que la cour a déjà adopté telle solution dans telle affaire. En d'autres termes, le précédent n'est pas une analogie parce qu'il est ressenti comme une contrainte, au même titre que la loi dont le juge se doit de faire application.

\section{La spécificité relative du raisonnement juridique}

Cette distinction reflète fort bien la finesse des analyses que recèle le livre de F. Schauer qui, là comme ailleurs, consiste en une enquête sur la spécificité du raisonnement juridique mais, disons le tout de suite, une enquête plus horizontale que verticale : F. Schauer cherche moins à convaincre de la spécificité du raisonnement juridique à l'aide d'une théorie propre qu'à mettre en lumière certains aspects du droit qui le distinguent de toutes les autres formes d'activités faisant elles aussi appel à des règles. Rien ne semble en effet plus difficile que de distinguer le raisonnement juridique de tout autre car le droit ou les juristes ne semblent pas faire appel à des raisonnements spécifiques auxquels n'auraient pas ou ne pourraient pas avoir recours d'autres disciplines ou d'autres professions. Quand bien même on voudrait retenir du raisonnement juridique qu'il est fondé sur des règles, ou qu'il procède à partir de règles, on serait bien en peine de lui trouver une originalité quelconque car beaucoup d'autres raisonnements reposent eux aussi sur des règles.

Pour autant, F. Schauer n'a jamais cessé de chercher à identifier la spécificité du raisonnement juridique, en entendant toutefois cette «spécificité » ou « unicité » dans un sens plutôt faible. Déjà, dans un article paru en 2003, il expliquait très bien ce qu'il entendait par la spécificité - somme toute modeste ou du moins faible - du raisonnement juridique :

The serious claims (which may still not be true...) that there really is something called legal reasoning are not claims that there is a totally unique form of thought dominant in law but non-existent elsewhere. Rather, the case for legal reasoning is that certain forms of reasoning - possibly reasoning from rules, possibly reasoning from precedent, possibly giving reasons, and possibly other forms of reasoning in which both authority and generalization loom large - are sufficiently more present in law than elsewhere that the claim of there being something worthy of being called legal reasoning is justified 6

6. Frederick Schauer, « Neutrality and Judicial Review », Law and Philosophy, 22 (3/4), 2003, p. 217-240 note 23. On trouvait déjà cette réflexion dans son ouvrage Playing by the Rules, op. cit. où il examinait la différence entre un précédent et une règle péremptoire (not. p. 182-187). De plus, la parenthèse qui fait

Texte paru dans Droit et Société, 2013/1, n. 83, p. 193-202 
Or, pour y revenir, raisonner à partir de précédent, c'est encore raisonner à partir d'une règle établie, que le "law maker » suit parce qu'il se sent tenu de le faire quand bien même cette règle le conduirait à adopter une solution qui lui semblerait « injuste ». De ce point de vue, la thèse de F. Schauer serait compatible avec celle de L. Alexander et E. Sherwin : raisonner à partir d'un précédent, c'est bien raisonner à partir d'une règle et procéder ainsi par déduction. L'analogie, elle, apparaît comme une technique de justification d'une solution. Lorsqu'il raisonne par analogie, le tribunal, ou le juge, conserve le choix de la source analogue et sélectionne une décision parmi un grand nombre de possibilités sur le fondement de ce qui lui semble le plus utile. La décision analogue est retenue en vue d'appuyer un argument tandis que, dans le précédent, une décision s'impose d'elle-même pour empêcher qu'une autre solution ne puisse être prise.

On comprend, à partir de cet exemple, que les deux ouvrages sont fortement ancrés dans le système de common law et peuvent paraître, pour cette raison même, quelque peu exotiques aux juristes formés dans le système de droit continental. Et si l'ouvrage de L. Alexander et $E$. Sherwin souffre très évidemment de rester cantonné dans un système, ce n'est pas du tout le cas de celui de F. Schauer. Approfondissant sa quête dans un chapitre très éclairant consacré justement au common law, il revient sur ce qui en fait la particularité, à savoir sa souplesse, son adaptabilité, sa flexibilité.

\section{Common law et droit civil}

Mais ces qualités n'en font pas pour autant un modèle que l'on pourrait opposer au modèle du droit civil codifié. À cet égard, conscient de l'enjeu politique voire géopolitique que la confrontation des modèles juridiques dissimule (assez mal d'ailleurs), $\mathrm{F}$. Schauer tord le cou à quelques clichés qui voudraient voir dans le common law aussi bien un droit entièrement fait par des juges qu'un droit purement et simplement déclaré par eux. De même, et inversement, il écorne la représentation naïve, mais fréquente outre-Atlantique, d'un droit civil rigide, sinon rigidifié dans le formalisme du code et qui transformerait les juges en des automates. Renvoyant dos à dos ces deux caricatures, il insiste sur le rapprochement que ces modèles connaissent aujourd'hui. II aurait d'ailleurs pu aller plus loin, mais ce n'était pas là son objet ni son sujet. II prend toutefois la peine de relever que ce prétendu "droit des juges », qui serait bien plus vivant que "le droit des codes ", n'est transmis aux étudiants des law schools que par des livres - les fameux case books - lesquels ne contiennent bien souvent qu'une succession de décisions - des cas - et d'opinions des juges eux-mêmes et fort peu de commentaires de ces décisions, ni même d'analyses critiques de ces mêmes décisions. Ainsi, les law schools forment-elles non pas tant à réfléchir sur le droit qu'à produire - reproduire ? - le droit tel qu'il se trouve dans les livres et entretiennent ou procèdent de l'idée que le droit s'apprend en lisant des

allusion à une thèse contraire renvoie à l'article de Larry ALEXANDER, "The Banality of Legal Reasoning », Notre Dame Law Review, 73, 1998, p. 517-533.

Texte paru dans Droit et Société, 2013/1, n. 83, p. 193-202 
opinions de juges ${ }^{7}$. Or, ces décisions, comme ces opinions, ne sont que des conclusions et non des points de départ : un cas ne se construit pas à partir d'une opinion qu'un juge pourra émettre sur la solution qui sera ensuite retenue. Au contraire, insiste F. Schauer, ce qui est central en common law c'est moins l'opinion des juges que le cas lui-même et donc la question litigieuse qui sera posée aux juges : c'est à partir d'elle que l'on peut mesurer la façon dont le juge « fera le droit », soit en prolongeant le droit existant, soit en le modifiant. Ainsi, la démarche première des juges sera toujours de s'interroger sur le point de savoir si telle question a déjà été jugée et, le cas échéant, comment. Mais on pourrait faire remarquer qu'il en va exactement de même dans un système de droit codifié : le problème est toujours de savoir si telle question de droit que soulève tel cas a déjà été jugée ou non.

Par ailleurs, F. Schauer revient sur les arguments pro et contra envers le common law en introduisant une dimension inédite.

On sait que le droit de common law a toujours suscité la critique de ceux qui y ont vu d'abord et avant tout un droit des juges se dissimulant eux-mêmes derrière l'idée qu'ils se contentaient de le « déclarer ». Hobbes, puis Bentham et Austin furent parmi les premiers et on se souvient de la formule cinglante de ce dernier dénonçant chez Blackstone: "The childish fiction employed by our judges, that judiciary or common law is not made by them, but is a miraculous something made by nobody, existing, I suppose, from eternity, and merely declared from time to time by the judges ${ }^{8}$. $»$

Néanmoins, F. Schauer fait remarquer que cette critique est relativement récente. Longtemps, il fut parfaitement admis que le common law était fait par les juges et que c'était là la meilleure façon de le faire car le droit pouvait se nourrir de l'expérience passée et s'ajuster aux situations, en évoluant progressivement et en pleine harmonie avec la société. Le présupposé implicite était - et est toujours - que chaque cas jugé isolément est représentatif d'autres cas et donc universalisable. Or, des travaux récents de psychologie ont permis de mettre en évidence une forme de raisonnement inconscient - l' « heuristique (ou stratégie) de la disponibilité 9 » - consistant à fonder une croyance sur une information immédiatement disponible, un exemple, plutôt que d'étayer cette information ou de compléter cet exemple par d'autres exemples pour vérifier la croyance. Or, à force de raisonner à partir d'exemples, les juristes de common law s'exposent précisément à ce risque consistant à nourrir la croyance que tel cas isolé - tel exemple - qui leur vient à l'esprit est plus représentatif qu'il ne l'est en réalité.

7. F. SCHAUER, p. 109: "In the traditional casebook, what comes after a case is not a series of questions or problems or comments but the next case, and then the next case and the next case after that. And traditionally, and still to a substantial extent, students are expected to learn the law by reading judicial opinions. "

8. John Austin, Lectures on Jurisprudence, Londres : J. Murray, $5^{\mathrm{e}}$ éd., 1885, vol. 2, p. 655.

9. Cf. Amos TVersky et Daniel Kahneman, « Availability: A Heuristic for Judging Frequency and Probability ", Cognitive Psychology, 5 (1), 1973, p. 207-233, qui ont mis en avant cette forme de raccourci mental inconscient fondé sur l'idée : " if you think of it, it must be important ». L. Alexander et E. Sherwin font eux aussi référence aux travaux de D. Kahneman mais ils n'utilisent pas le concept d'heuristique de la disponibilité. Voir aussi Thomas Gilovich, Dale Griffin et Daniel Kahneman (eds.), Heuristics and Biases. The Psychology of Intuitive Judgement, Cambridge : Cambridge University Press, 2002.

Texte paru dans Droit et Société, 2013/1, n. 83, p. 193-202 
II reste que le common law dispose de cette faculté à évoluer et changer en fonction de situations, en s'adaptant " par lui-même » au cas par cas comme tendent à le penser ses partisans. D'où sa grande flexibilité qui résulte, selon $F$. Schauer, de ce que les règles de common law changent au moment même où elles s'appliquent. Mais comment changent-elles? Tout simplement en subissant un tempérament, une restriction de la part des juges, lesquels disposent du pouvoir d'ajouter une exception à la règle préalablement admise comme existante par tous. Et pourquoi ? Parce qu'ils l'estiment nécessaire au regard de l'équité.

\section{Le common law, la rétroactivité et les principes de Dworkin}

Ainsi retrouve-t-on une autre des critiques ou objections les plus fréquemment opposées au common law et sur laquelle Bentham fut un des premiers à insister : sa rétroactivité. À cet égard, F. Schauer relève, presque en passant, que certains, dont Ronald Dworkin, ont tenté de surmonter l'objection en expliquant que le common law ne change pas. L'argument de Dworkin est désormais bien connu : ce que l'on tend à prendre pour des règles - sous l'influence, d'après lui néfaste, du positivisme sont en réalité des principes qui existent avant que le cas n'arrive devant le juge et avant même que les juges n'en fassent application. Ce qui semble être un changement dans le droit est en fait la démonstration de ce que, lorsqu'une cour recherche sérieusement la bonne réponse que le cas appelle, elle finit par révéler celle qui existe plutôt que d'en créer une nouvelle ${ }^{10}$.

Cette thèse de Dworkin a été fortement contestée, notamment par Kenneth Kress 11 et Frederick Schauer n'y revient pas. On peut presque le regretter. En effet, si le texte de Kress est certainement une des meilleures critiques internes jamais écrites contre Dworkin (qui en vit bien d'autres et sut feindre d'y répondre pour toujours mieux s'y soustraire), il omet de souligner une des fonctions implicites mais très évidemment nécessaires de la distinction entre les règles et les principes: celle d'introduire dans le droit positif sublunaire une temporalité radicalement différente, d'une densité ou d'une épaisseur, pourrait-on dire, sur laquelle nul ne peut jamais avoir la moindre prise. Et c'est précisément là sa fonction. Au fond, les principes de Dworkin bénéficient de la même intemporalité que la figure d'un dieu. Et de même qu'il n'y a aucun sens, pour le croyant, à opposer le temps historique, sublunaire et humain, à un dieu, il n'y a aucune pertinence à demander, du point de vue de Dworkin, quand est apparu un principe. La seule difficulté, pourtant, est que la thèse de l'existence de principes relève bien d'une forme de croyance ou de foi. Elle est donc logiquement indéfendable.

Aussi étrange que cela puisse paraître, il en va de même avec la thèse de la souplesse et/ou de la flexibilité du common law : ceux qui y croient le diront souple et flexible pour ne pas avoir à s'interroger sur les modalités de cette même flexibilité. II faut d'ailleurs souligner à quel point les arguments exposés par F. Schauer pour la

10. Voir Frederick Schauer, p. 115.

11. Kenneth J. KRESS, «Legal Reasoning and Coherence Theories: Dworkin's Rights Thesis, Retroactivity, and the Linear Order of Decisions », California Law Review, 72 (3), 1984, p. 369.

Texte paru dans Droit et Société, 2013/1, n. 83, p. 193-202 
défendre sont à double tranchant et rejoignent, à bien des égards, ceux mobilisés par L. Alexander et E. Sherwin dans leur entreprise de démystification. On s'étonne cependant que ces derniers n'en profitent pas, sinon pour repenser un peu la pédagogie des law schools, du moins pour s'interroger sur le rôle qu'elles jouent dans la mystification du raisonnement juridique. Ils suggèrent certes de renforcer l'enseignement de la logique et des méthodes empiriques, tout en se gardant de définir ce qu'ils entendent par là, mais c'est en passant. Et ils prêtent alors peut-être le flanc à la critique bien connue de Holmes selon laquelle le droit n'est pas une affaire de logique mais d'expérience. Enfin, en s'attachant comme ils le font à l'idée que l'application d'une règle requiert, de la part de celui qui l'applique, qu'il se fie à l'intention de l'auteur de la règle 12 , ils semblent substituer un mythe (celui de l'intention de l'auteur) à un autre et l'on ne peut s'empêcher de conclure que l'entreprise de démystification manque quelque peu de systématicité voire de profondeur.

\section{Le réalisme, son héritage et ses limites}

Le livre de F. Schauer n'est lui non plus guère novateur sur la question de l'interprétation textuelle mais il contient, en revanche, des développements très précieux sur la stratégie du juriste d'aujourd'hui confronté à l'héritage sceptique de Holmes et des Réalistes. En effet, quand bien même Holmes et ses héritiers eurent raison de penser, à l'image du Chief Justice Charles Evans Hughes (qui n'était cependant pas connu pour être réaliste) que « la constitution est ce que les juges disent qu'elle est " 13 , il n'en demeure pas moins que les cours et les tribunaux ne se contentent pas d'argumentations qui s'en remettent à leur propre pouvoir. Mieux encore, ils sont bien souvent tentés de les écarter d'un revers de la main au profit de ce que l'on appelle en anglais la « legal doctrine », qu'il ne faut pas confondre avec notre " doctrine juridique », et qui recouvre les catégories juridiques du droit en vigueur. On se trouve donc pris dans un jeu de dupes pour le moins paradoxal (mais somme toute très classique) : d'un côté, les juristes praticiens, et en premier lieu les avocats, ont l'impérieux besoin de construire des argumentations qui soient le plus « juridiques » possible ; de l'autre, le New Legal Realism ${ }^{14}$ d'aujourd'hui parvient à

12. Voir not. chap. 5.

13. "We are under a Constitution, but the Constitution is what the judges say it is », Charles Evans HUGHES [Governor of New York, York], "Speech before the Elmira Chamber of Commerce (May 3, 1907) ", in ID., Addresses of Charles Evans Hughes, 1906-1916, New York: G. P. Putnam's sons, $2^{\mathrm{e}}$ éd., 1916, p. 179, ici p. 185, cité par F. Schauer, p. 143, note 38. Précisons cependant que Hughes ajoute aussitôt: " and the judiciary is the safeguard of our liberty and of our property under the Constitution " et "I do not want to see any direct assault upon the courts, nor do I want to see any indirect assault upon the courts ». Son affirmation ne doit donc pas s'entendre comme une marque de défiance à l'égard des juges.

14. L'expression désigne ce courant au carrefour du droit et de la science politique qui se propose d'étudier empiriquement les modes de décisions des juridictions. Elle fut employée pour la première fois par Frank B. Cross, "Political Science and the New Legal Realism: A Case of Unfortunate Interdisciplinary Ignorance », Northwestern University Law Review, 92 (1), 1997, p. 251-326 et voir Frank B. CRoss et Stefanie A. LINDQUIST, "The Scientific Study of Judicial Activism », Minnesota Law Review, 91 (6), 2007, p. 1752 1784. Voir aussi Howard S. ERLANGer et al., "Is it Time for a New Legal Realism? », Wisconsin Law Review, 2, 2005, p. 335-363 ; Stewart MACAULAY, « The New Versus The Old Legal Realism: "Things Ain't What They Used To Be" », Wisconsin Law Review, 2, 2005, p. 365 ; Arthur F. MAcEvoY, "A New Realism For Legal Studies », Wisconsin Law Review, 2, 2005, p. 433 ; Thomas J. MILES et Cass R. SunStEIN, « The New Legal

Texte paru dans Droit et Société, 2013/1, n. 83, p. 193-202 
démontrer, preuves empiriques à l'appui, que les thèses des Réalistes d'hier sont pertinentes et que l'idéologie (lato sensu et pas seulement au sens politique) des juges détermine autant - sinon plus - que les règles de droit la solution du cas.

La résolution du paradoxe est ici non pas une question de logique mais de connaissance empirique et de savoir-faire. Pour penser comme un juriste (ou comme un avocat), il faut non seulement connaître les arguments auxquels sont sensibles les juges mais aussi savoir les dissimuler derrière des règles de droit qui parviendront à justifier telle solution plutôt que telle autre. Ainsi, même le plus réaliste des juristes a besoin de connaître le droit et de tenir compte des méthodes les plus classiques avec lesquelles les juges parviennent à justifier les règles qu'ils font. En d'autres termes, le droit n'est pas qu'un langage obéissant à certaines règles logiques, il est aussi une langue qu'il faut apprendre à parler pour se faire comprendre de ses locuteurs. Et cela vaut aussi bien pour le juriste non praticien lequel risque, s'il n'y prend pas garde, de ne voir que la moitié du problème ou une seule des deux faces de la médaille.

\section{L'auteur}

Professeur à l'Université Paris Ouest Nanterre-La Défense, Pierre Brunet est directeur du Centre de théorie et analyse du droit (UMR CNRS 7074) et co-directeur de l'École doctorale Sciences juridiques et politiques (ED 141). Membre de I'Institut universitaire de France (2009), il consacre la plupart de ses recherches à une approche analytique et réaliste du droit en général et du droit public en particulier. Ses travaux actuels portent sur les modes de production des concepts juridiques et les interactions de systèmes normatifs.

II a notamment publié :

— « Le raisonnement juridique : une pratique spécifique ? », International Journal for the Semiotics of Law - Revue internationale de sémiotique juridique (en ligne, 3 février 2013 et version imprimée à paraître) :

- "Le droit est-il est dans la tête ? (À propos d'un livre de Karl Olivecrona) », Jus Politicum, 8, 2012 (revue en ligne) ;

- Questions contemporaines de théorie analytique du droit (dir., avec Federico J. ARENA), Madrid : Marcial Pons, 2011.

Realism », University of Chicago Law Review, 75 (?), 2008, p. 81 ; Victoria NouRSE et Gregory SHAFFER, «Varieties of New Legal Realism. Can A New World Order Prompt A New Legal Theory? », Cornell Law Review, 95, 2009, p. 61.

Texte paru dans Droit et Société, 2013/1, n. 83, p. 193-202 